Check for updates

Cite this: Chem. Commun., 2018, 54,7854

Received 16th April 2018, Accepted 12th June 2018

DOI: $10.1039 / \mathrm{c} 8 \mathrm{cc} 03002 \mathrm{~b}$

rsc.li/chemcomm

\section{Kinetic barriers to $\alpha$-synuclein protofilament formation and conversion into mature fibrils $\dagger$}

\author{
James W. P. Brown, (D) $\ddagger^{a}$ Georg Meisl, (D) a Tuomas P. J. Knowles, (D) ab \\ Alexander K. Buell, (D) Christopher M. Dobson (D) *a and Céline Galvagnion (D) *ad
}

\begin{abstract}
Oligomeric and protofibrillar aggregates that are populated along the pathway of amyloid fibril formation appear generally to be more toxic than the mature fibrillar state. In particular, $\alpha$-synuclein, the protein associated with Parkinson's disease, forms kinetically trapped protofibrils in the presence of lipid vesicles. Here, we show that lipid-induced $\alpha$-synuclein protofibrils can convert rapidly to mature fibrils at higher temperatures. Furthermore, we find that $\beta$-synuclein, generally considered less aggregation prone than $\alpha$-synuclein, forms protofibrils at higher temperatures. These findings highlight the importance of energy barriers in controlling the de novo formation and conversion of amyloid fibrils.
\end{abstract}

The misfolding and aggregation of normally soluble proteins into insoluble amyloid deposits is associated with a wide range of devastating neurodegenerative diseases. ${ }^{1-3}$ Amyloidogenic proteins, including the amyloid- $\beta$-peptide (A $\beta 42)$ and $\alpha$-synuclein, the aggregation of which has been associated with Alzheimer's and Parkinson's diseases, respectively, have been found to populate a range of intermediate species prior to the formation of mature amyloid fibrils. ${ }^{4}$ These intermediate species include oligomers and protofibrils, and are proposed to be more toxic than mature fibrils and to induce the disruption of cell membranes. ${ }^{4,5}$

Unlike $A \beta 42, \alpha$-synuclein is intrinsically highly kinetically stable in its monomeric form in solution, in the absence of existing aggregates, ${ }^{6,7}$ suggesting a high free energy barrier that determines the rate of the homogeneous primary nucleation step.

\footnotetext{
${ }^{a}$ Centre for Misfolding Diseases, Department of Chemistry, University of Cambridge, Lensfield Road, Cambridge CB2 1EW, UK.

E-mail: celine.galvagnion@dzne.de, cmd44@cam.ac.uk; Tel:+44 (0)1223763070

${ }^{b}$ Cavendish Laboratory, Department of Physics, University of Cambridge,

JJ Thomson Avenue, Cambridge, CB3 $1 \mathrm{HE}, \mathrm{UK}$

${ }^{c}$ University of Düsseldorf, Institute of Physical Biology, Universitätsstr.1, 40225, Düsseldorf, Germany

${ }^{d}$ German Center for Neurodegenerative Diseases (DZNE), Sigmund-Freud-Str. 27, 53127, Bonn, Germany

$\dagger$ Electronic supplementary information (ESI) available: Materials and methods, supplementary figure. See DOI: 10.1039/c8cc03002b

\$ Present address: EMBL Australia Node in Single Molecule Science, University of New South Wales, Kensington, NSW 2052, Australia.
}

Under quiescent conditions, for example, no fibril formation can be detected by Thioflavin-T (ThT) fluorescence even when $\alpha$-synuclein is incubated at concentrations above physiological levels (40-45 $\mu \mathrm{M}$ in synaptic terminals ${ }^{8}$ ) and well above the critical aggregation concentration (0.7-2.7 $\mu \mathrm{M}$ in solution $\left.{ }^{9,10}\right)$ for 5 days. $^{7}$ The presence of specific interfaces, however, such as air/water, ${ }^{11}$ polystyrene/water, ${ }^{12,13}$ detergent/water ${ }^{14,15}$ and lipid/water ${ }^{7,12,16}$ have been found to induce the rapid aggregation of $\alpha$-synuclein, and indeed mechanical agitation is often used in experimental studies and can result in observable fibril formation within hours. ${ }^{12,17,18}$ Under well controlled solution conditions, a number of specific aggregated species ranging from spherical oligomers $^{19}$ to lipid-induced protofibrils ${ }^{7}$ could be studied in their kinetically trapped states. In the latter example, negatively charged model membranes have been shown to induce the aggregation of $\alpha$-synuclein into protofibrillar structures which appear to not convert further at a detectable rate even in the presence of high micromolar concentrations of soluble $\alpha$-synuclein. ${ }^{7}$ However, these protofibrillar structures were found to convert into mature fibrils after sonication ${ }^{7}$ or through secondary processes. $^{20}$

Protofibrils, which are morphologically distinguished from mature amyloid fibrils, have been shown to have distinct kinetic properties, including a reduced tendency to act as templates for further growth. ${ }^{21,22}$ The kinetics of conversion from protofibrillar to fibrillar species of $\alpha$-synuclein have not yet been well characterised and, as in other systems, ${ }^{22}$ this may be a critical step in the self-replication and proliferation of aggregates.

In the present study, we explore the conditions for the aggregation of $\alpha$-synuclein in the presence of lipid membranes in order to investigate the conditions under which the lipidinduced protofibrils are able to convert into mature fibrils such as those observed under shaking conditions in aqueous solution. In particular, we incubated $\alpha$-synuclein in the presence of small unilamellar vesicles (SUV) prepared with 1,2-dimyristoyl-snglycero-3-phospho-L-serine (DMPS) at temperatures ranging from $30{ }^{\circ} \mathrm{C}$ (Fig. 1a) to $60{ }^{\circ} \mathrm{C}$ (Fig. 1b). In agreement with previous results, ${ }^{7,23-25}$ monomeric $\alpha$-synuclein readily forms ThT-positive 
a
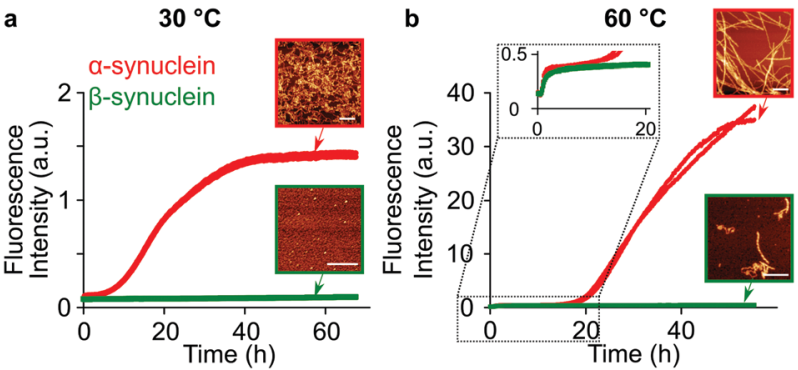

Fig. 1 Lipid-induced aggregation of $\alpha$-synuclein and $\beta$-synuclein at 30 and $60{ }^{\circ} \mathrm{C}$. (a and b) Changes in ThT fluorescence when $100 \mu \mathrm{M} \alpha$-synuclein (red) or $\beta$-synuclein (green) were incubated in the presence of $100 \mu \mathrm{M}$ DMPS under quiescent conditions at $\mathrm{pH} 6.5$ and $30{ }^{\circ} \mathrm{C}$ (a) or $60^{\circ} \mathrm{C}$ (b). The scale bars on the AFM images correspond to $500 \mathrm{~nm}$.

protofibrillar structures at $30{ }^{\circ} \mathrm{C}$ (Fig. 1a, upper inset). These protofibrils have a diameter of $3.8 \pm 0.3 \mathrm{~nm}$, as determined from the height profiles obtained from Atomic Force Microscopy (AFM) images in air, and an average length of $167 \pm 62 \mathrm{~nm} .^{7}$ At $60{ }^{\circ} \mathrm{C}$ (Fig. 1b), however, we observed a biphasic increase in the ThT-fluorescence intensity, characterised by the presence of two well defined plateau regions and AFM images of samples taken at the second plateau phase reveal the presence of mature $\alpha$-synuclein fibrils, with a diameter of $8.5 \pm 1.9 \mathrm{~nm}$ and a greatly increased average length $(1.5 \pm 0.5 \mu \mathrm{m})$. These results show that an increase in the temperature induces a conversion of lipid-induced $\alpha$-synuclein protofibrils into mature fibrils.

Interestingly, such an increase in temperature also led to the formation of a mixture of lipid-induced protofibrils and mature fibrils by $\beta$-synuclein, a homologous protein of $\alpha$-synuclein. In vivo, $\beta$-synuclein is expressed at the same location (e.g. synaptic termini) and at similar levels as $\alpha$-synuclein ${ }^{8}$ but it has a lower propensity to aggregate both in vivo and in vitro. $\beta$-synuclein has been shown to form proteinase K-resistant aggregates in dopaminergic neurons in rats ${ }^{26}$ but reproducing fibril formation under controlled conditions in vitro has remained challenging. Under shaking conditions, the presence of metals $\left(\mathrm{Zn}^{2+}, \mathrm{Pb}^{2+}\right.$ and $\left.\mathrm{Cu}^{2+}\right),{ }^{27}$ SDS micelles ${ }^{28}$ or mildly acidic $\mathrm{pH}(5.8),{ }^{29}$ has, however, been found to induce the aggregation of $\beta$-synuclein. Our results show that an increase in temperature is sufficient to overcome the high kinetic barrier to the formation of amyloid fibrils by $\beta$-synuclein in the presence of SUV but in the absence of metals, micelles or acidic $\mathrm{pH}$. Unlike $\alpha$-synuclein, $\beta$-synuclein was found to form a mixture of protofibrils and mature fibrils at $60{ }^{\circ} \mathrm{C}$ and therefore not to have reached the equilibrium state. As a result, we chose to investigate in more detail the influence of increasing temperatures on the mechanism of lipid-induced aggregation of $\alpha$-synuclein only, as this system reaches equilibrium within the time scale of our experiments at $60{ }^{\circ} \mathrm{C}$.

First, we investigated the effects of changes in both the protein (Fig. 2a, c and e) and lipid concentrations (Fig. 2b, $\mathrm{d}$ and $\mathrm{f}$ ) on the rate of amyloid fibril formation by $\alpha$-synuclein (Fig. 2c and d) as well as on the quantities of fibrils that formed at $50{ }^{\circ} \mathrm{C}$ (Fig. 2e and f). This temperature was chosen because it allows a more quantitative analysis of the aggregation mixture at the end of the experiments, as sample evaporation is reduced
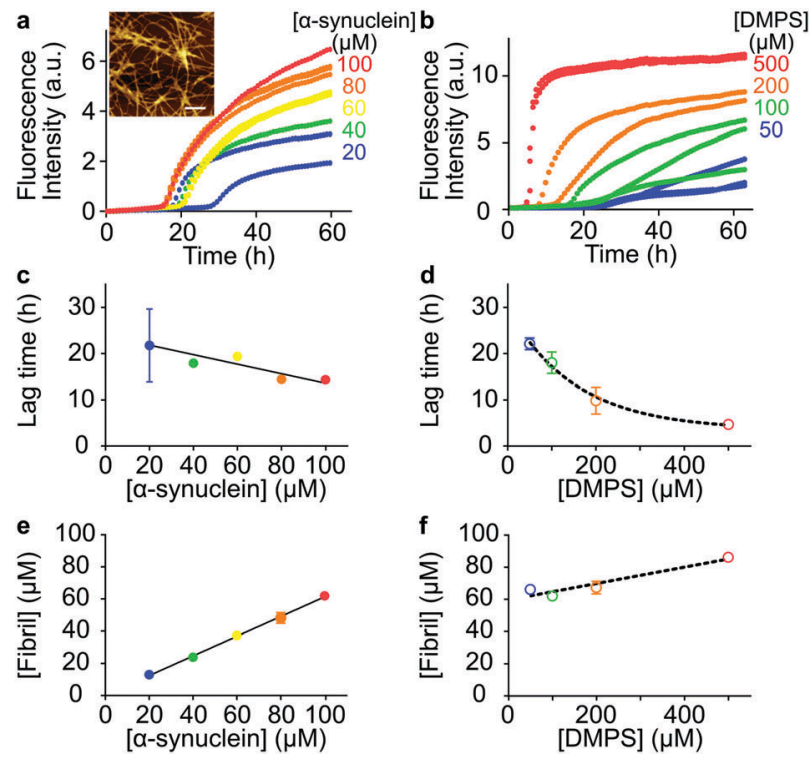

Fig. 2 Characterisation of $\alpha$-synuclein aggregation kinetics in the presence of DMPS at $50{ }^{\circ} \mathrm{C}$. (a and b) Changes in ThT fluorescence when increasing concentrations of $\alpha$-synuclein were incubated in the presence of $100 \mu \mathrm{M}$ DMPS (a) or when $100 \mu \mathrm{M} \alpha$-synuclein was incubated in the presence of increasing concentrations of DMPS (b). (c-f) Changes in the lag times of the reaction of amyloid formation ( $c$ and $d$ ) or the concentration of fibrils formed after $60 \mathrm{~h}$ (e and f) with increasing concentrations of $\alpha$-synuclein (c and e) or increasing concentrations of DMPS ( $d$ and $f$ ).

relative to higher temperatures. The lag-time of the second, large increase in $\mathrm{ThT}$ fluorescence of the aggregation reaction was found to decrease to a small extent with increasing initial concentrations of $\alpha$-synuclein monomer (Fig. 2c) but to larger extent with increasing initial concentrations of DMPS (Fig. 2d). The final concentrations of fibrils, determined using UV absorbance measurements (see ESI $\dagger$ for more details), were observed to increase strongly with increasing initial concentrations of soluble $\alpha$-synuclein (Fig. 2e) but less so with the initial concentrations of DMPS (Fig. 2f).

AFM analysis of the reaction mixture at the end of the aggregation reaction shows that the major species that $\alpha$-synuclein forms at this temperature are amyloid fibrils of $2.0 \pm 0.8 \mu \mathrm{m}$ in length and $5.6 \pm 0.3 \mathrm{~nm}$ in height (Fig. 2a (inset) and Fig. S1, ESI $\dagger$ ). The fibrils formed at $50{ }^{\circ} \mathrm{C}$ are therefore intermediate in thickness between those formed at $30{ }^{\circ} \mathrm{C}$ and those formed at $60{ }^{\circ} \mathrm{C}$. In what follows, we refer to the two types of fibrils mentioned above as "protofibrils" for the thinnest curly fibrils formed at $30^{\circ} \mathrm{C}$ (see inset Fig. 1a, AFM image in red square) and "mature fibrils" for all significantly thicker fibrils formed at temperatures of $50{ }^{\circ} \mathrm{C}$ and above (see inset Fig. 1b, AFM image in red square and inset Fig. 2a). Overall, these results suggest that the process of lipid-induced protofibril formation differs from that of the lipid-induced mature fibril formation. In particular, we find that the determining factor of mature fibril mass at the end of the reaction is the initial concentration of monomeric protein, as observed for most amyloid proteins, ${ }^{10}$ whereas it is the concentration of DMPS vesicles in the case of protofibril formation. ${ }^{7}$ Moreover, our results suggest the possibility that, under quiescent conditions, an increase in temperature is 


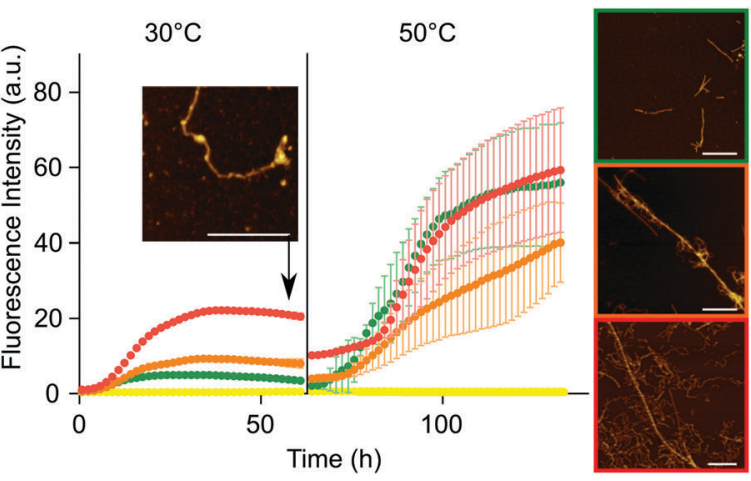

Fig. 3 Aggregation of $\alpha$-synuclein in the presence of DMPS vesicles measured using a temperature jump from $30{ }^{\circ} \mathrm{C}$ to $50{ }^{\circ} \mathrm{C}$. Changes in ThT fluorescence when $100 \mu \mathrm{M} \alpha$-synuclein was incubated in the presence of 0 (yellow), 100 (green), 200 (orange) or $500 \mu \mathrm{M}$ DMPS (red) under quiescent conditions at pH 6.5. The solutions were first incubated at $30{ }^{\circ} \mathrm{C}$ until the ThT fluorescence reached a plateau, and then the temperature was increased to $50{ }^{\circ} \mathrm{C}$. The AFM images show the morphology of the fibrils formed at the plateau phase at $30^{\circ} \mathrm{C}$ (left) and at $50{ }^{\circ} \mathrm{C}$ (right) and correspond to the reaction mixtures when $100 \mu \mathrm{M}$ $\alpha$-synuclein was incubated in the presence of 100 (top), 200 (middle) or $500 \mu \mathrm{M}$ DMPS (bottom). The scale bars on the AFM images correspond to $500 \mathrm{~nm}$.

already sufficient to overcome the activation energy for the formation of mature fibrils and that mechanical action is not necessarily required.

We used sequential temperature jump experiments to investigate whether or not there is direct conversion between $\alpha$-synuclein protofibrils and mature fibrils along the aggregation time course. These aggregation experiments were performed at $30{ }^{\circ} \mathrm{C}$ until the ThTfluorescence reaches a plateau, at which point the temperature was increased to $50{ }^{\circ} \mathrm{C}$ (Fig. 3). AFM analysis of the reaction mixture at the plateau phase at $30{ }^{\circ} \mathrm{C}$, before the change of temperature, shows the presence of protofibrils (Fig. 3) with a diameter of $3.8 \pm 0.3 \mathrm{~nm}$ (as calculated from AFM height profiles). At this stage, 10 (green curve Fig. 3), 20 (orange curve Fig. 3) and 50 (red curve Fig. 3) $\mu \mathrm{M}$ monomeric protein had been converted into protofibrils in the presence of 100, 200 and $500 \mu \mathrm{M}$ DMPS, respectively, as determined using UV absorbance measurements (see $\mathrm{ESI} \dagger$ for more details). When the temperature was switched to $50{ }^{\circ} \mathrm{C}$, the value of ThTfluorescence first decreased, a process that we attributed to a change in the ThT quantum yield due to the change in temperature, ${ }^{30}$ and then increased very substantially until it reached a second plateau (Fig. 3). At this plateau phase, the fibril concentration reached a value of $80 \mu \mathrm{M}$ for the different initial DMPS concentrations. AFM analysis of the reaction mixture at this second plateau phase shows the presence only of mature fibrils of several microns in length and a diameter of $5.6 \pm 0.7 \mathrm{~nm}$. The ThT fluorescence intensity in the presence of protofibrillar species at $50{ }^{\circ} \mathrm{C}$, i.e. right after the temperature increase, was found to be $100 \pm 19$ units per $\mu \mathrm{M}$ of fibril in our particular fluorescence experiment, while ThT bound to mature fibrillar species formed over time had a fluorescence intensity of $564 \pm 115$ units per $\mu \mathrm{M}$ of fibrils (as measured under identical experimental conditions). We observed a similar increase in the intensity of the ThT fluorescence when the reaction mixture evolved from lipid-induced protofibrils to mature fibrils in the case of the C-truncated variant AS $1-103 .^{20}$ This change in ThT fluorescence efficiency may be attributable to a change in the bound conformation or fluorescence quantum yield of $\mathrm{ThT}^{30}$ due to fibril maturation, which could be linked to a higher degree of ordering in the mature fibrils or enhanced higher order assembly of fibrils. ${ }^{31} \alpha$-synuclein was not found to form ThT-active fibrillar species when incubated in the absence of DMPS SUVs at 30 and $50{ }^{\circ} \mathrm{C}$ (yellow curve Fig. 3), confirming that the presence of lipid-induced protofibrils is required for the formation of mature fibrils under these conditions (quiescent and non-binding plate surfaces).

The observation that, even at elevated temperatures, the aggregation curves of $\alpha$-synuclein in the presence of lipids show biphasic behaviour prompted us to probe the temperature dependence of the initial step, the formation of protofibrils. We studied the kinetics of the initial phase of the lipid-induced aggregation, which includes both primary nucleation and elongation of $\alpha$-synuclein protofibrils, at temperatures ranging from 25 to $60{ }^{\circ} \mathrm{C}$ (Fig. 4a). We observed that the lag phase decreases with increasing temperature. In order to obtain quantitative information on the kinetic barriers associated with the lipid-induced protofibril formation, we analysed the early times of the aggregation curves using a one-step nucleation model, as described previously. ${ }^{7,25}$ In particular, we carried out a global fit of our kinetic data measured at temperatures ranging from 25 to $60{ }^{\circ} \mathrm{C}$ using one free parameter, $k_{n} k_{+}$, the combined rate constants of the nucleation and elongation steps (inset Fig. 4a). Using the values of $k_{n} k_{+}$ determined from our fits, we then determined the activation
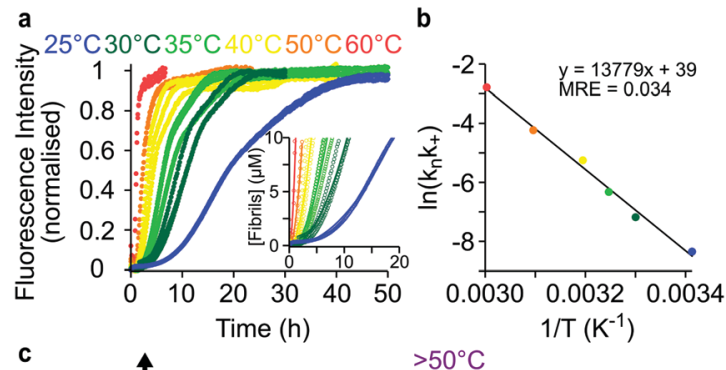

C

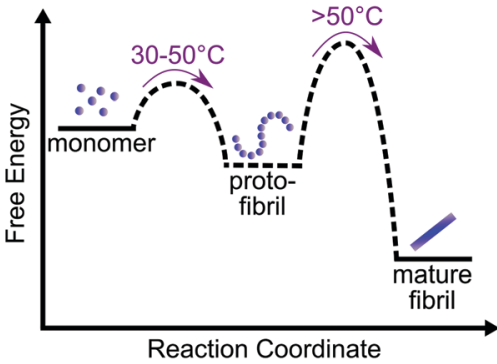

Fig. 4 Temperature dependence of $\alpha$-synuclein lipid-induced protofibril formation. (a) Change in the ThT fluorescence when $100 \mu \mathrm{M}$ free $\alpha$-synuclein were incubated in the presence of $100 \mu \mathrm{M}$ DMPS SUVs under quiescent conditions at $\mathrm{pH} 6.5$ and at temperatures ranging from 20 to $60{ }^{\circ} \mathrm{C}$. Data are normalised to initial plateau value. Inset: Fit of the early time points of the aggregation curves to a one-step nucleation model ${ }^{7}$ using AmyloFit ${ }^{33}$ with $n=0.25$ and $K_{M}=125 \mu \mathrm{M}$ (as determined previously ${ }^{7,24,25}$ ) (MRE $=5 \times$ $\left.10^{-13} \mathrm{M}^{-2}\right)$. (b) Plot of $\ln \left(k_{n} k_{+}\right)$against $\frac{1}{T}$. The data were fitted to a linear equation using AmyloFit. ${ }^{33}$ (c) Schematic free energy diagram for the conversion of monomeric $\alpha$-synuclein to mature fibrils suggested by the data in the present study. 
energy, $E_{\mathrm{a}}$, of the combined primary nucleation and elongation reaction using the Arrhenius equation, $k_{n} k_{+}=A \mathrm{e}^{\frac{-E_{\mathrm{a}}}{R T}}$, where $A$ is the pre-exponential factor, $R$ the gas constant and $T$ the absolute temperature in Kelvin (K). The plot of $\ln \left(k_{n} k_{+}\right)$versus $1 / T$ gives a slope equal to $\frac{-E_{\mathrm{a}}}{R}$, which was then used to determine the sum of the Arrhenius activation energies of lipid-induced nucleation and elongation, $E_{\mathrm{A}}=E_{\mathrm{A}}^{\mathrm{Nuc}}+E_{\mathrm{A}}^{\mathrm{El}}$, and hence approximately the corresponding activation enthalpy, $E_{\mathrm{a}} \approx \Delta H^{\ddagger}$ of $115 \pm 3 \mathrm{~kJ} \mathrm{~mol}^{-1}$. This value for the combined barrier for nucleation and growth is approximately twice the value of that determined for the elongation of mature fibrils from seeded experiments. ${ }^{6,32}$ It is interesting to note that the nucleation step appears to involve an enthalpic barrier similar in magnitude to that for the elongation step, despite the fact that the nucleation step is likely to involve a substantial change in secondary structure, from the $\alpha$-helical membrane-bound state $^{7}$ to the $\beta$-sheet rich amyloid state. It has been reported previously that the enthalpic part of the free energy barrier for fibril growth correlates with the extent of secondary structure in the monomeric precursor, ${ }^{32}$ a finding that is likely to apply also to the nucleation step, with the addition that the structural rearrangement needs to occur in more than one molecule simultaneously.

Characterising the kinetic barriers for the individual microscopic processes involved in amyloid formation, and their dependence on a range of parameters, is crucial in developing a fundamental understanding of how $\alpha$-synuclein aggregates in disease. In this study, we have described a temperature dependent switch from protofibrils, the formation of which is limited by the quantity of lipids added to the system, ${ }^{7}$ to a more mature state in which the extent of fibril formation is governed by the total concentration of protein. We propose that the temperature change enables the $\alpha$-synuclein-lipid system to convert from the metastable, protofibrillar state and overcome the kinetic barrier corresponding to the formation of mature fibrils. Our study provides insights into the basic mechanisms of lipid-induced fibril formation by $\alpha$-synuclein and elucidates the relationship between protofibrils and mature fibrils.

\section{Conflicts of interest}

There are no conflicts to declare.

\section{References}

1 F. Chiti and C. M. Dobson, Annu. Rev. Biochem., 2006, 75, 333-366. 2 T. P. J. Knowles, M. Vendruscolo and C. M. Dobson, Nat. Rev. Mol. Cell Biol., 2014, 15, 384-396.

3 F. Chiti and C. M. Dobson, Annu. Rev. Biochem., 2017, 86, 27-68.

4 B. Caughey and P. T. Lansbury, Annu. Rev. Neurosci., 2003, 26, 267-298.

5 G. Fusco, S. W. Chen, P. T. F. Williamson, R. Cascella, M. Perni, J. A. Jarvis, C. Cecchi, M. Vendruscolo, F. Chiti, N. Cremades, L. Ying, C. M. Dobson and A. De Simone, Science, 2017, 358, 1440-1443.
6 A. K. Buell, C. Galvagnion, R. Gaspar, E. Sparr, M. Vendruscolo, T. P. J. Knowles, S. Linse and C. M. Dobson, Proc. Natl. Acad. Sci. U. S. A., 2014, 111, 7671-7676.

7 C. Galvagnion, A. K. Buell, G. Meisl, T. C. T. Michaels, M. Vendruscolo, T. P. J. Knowles and C. M. Dobson, Nat. Chem. Biol., 2015, 11, 229-234. 8 B. G. Wilhelm, S. Mandad, S. Truckenbrodt, K. Kröhnert, C. Schäfer, B. Rammner, S. J. Koo, G. A. Claßen, M. Krauss, V. Haucke, H. Utrlaub and S. O. Rizzoli, Science, 2014, 344, 1023-1028.

9 M. Iljina, G. A. Garcia, M. H. Horrocks, L. Tosatto, M. L. Choi, K. A. Ganzinger, A. Y. Abramov, S. Gandhi, N. W. Wood, N. Cremades, C. M. Dobson, T. P. J. Knowles and D. Klenerman, Proc. Natl. Acad. Sci. U. S. A., 2016, 113, E1206-E1215.

10 A. J. Baldwin, T. P. J. Knowles, G. G. Tartaglia, A. W. Fitzpatrick, G. L. Devlin, S. L. Shammas, C. A. Waudby, M. F. Mossuto, S. Meehan, S. L. Gras, J. Christodoulou, S. J. Anthony-Cahill, P. D. Barker, M. Vendruscolo and C. M. Dobson, J. Am. Chem. Soc., 2011, 133, 14160-14163.

11 S. Campioni, G. Carret, S. Jordens, L. Nicoud, R. Mezzenga and R. Riek, J. Am. Chem. Soc., 2014, 136, 2866-2875.

12 M. Grey, S. Linse, H. Nilsson, P. Brundin and E. Sparr, J. Parkinson's Dis., 2011, 1, 359-371.

13 R. Vácha, S. Linse and M. Lund, J. Am. Chem. Soc., 2014, 136, 11776-11782.

14 L. Giehm, C. L. P. Oliveira, G. Christiansen, J. S. Pedersen and D. E. Otzen, J. Mol. Biol., 2010, 401, 115-133.

15 R. C. Rivers, J. R. Kumita, G. G. Tartaglia, M. M. Dedmon, A. Pawar, M. Vendruscolo, C. M. Dobson and J. Christodoulou, Protein Sci., 2008, 17, 887-898.

16 M. Rabe, A. Soragni, N. P. Reynolds, D. Verdes, E. Liverani, R. Riek and S. Seeger, ACS Chem. Neurosci., 2013, 4, 408-417.

17 V. N. Uversky, J. Li, P. Souillac, I. S. Millett, S. Doniach, R. Jakes, M. Goedert and A. L. Fink, J. Biol. Chem., 2002, 277, 11970-11978.

18 D. Bhattacharyya, R. Kumar, S. Mehra, A. Ghosh, S. K. Maji and A. Bhunia, Chem. Commun., 2018, 54, 3605-3608.

19 N. Lorenzen, S. B. Nielsen, A. K. Buell, J. D. Kaspersen, P. Arosio, B. S. Vad, W. Paslawski, G. Christiansen, Z. Valnickova-Hansen, M. Andreasen, J. J. Enghild, J. S. Pedersen, C. M. Dobson, T. P. J. Knowles and D. E. Otzen, J. Am. Chem. Soc., 2014, 136, 3859-3868.

20 I. M. van der Wateren, T. P. Knowles, A. K. Buell, C. M. Dobson and C. Galvagnion, Chem. Sci., 2018, DOI: 10.1039/C8SC01109E.

21 J. D. Harper, S. S. Wong, C. M. Lieber and P. T. Lansbury, Chem. Biol., 1997, 4, 119-125.

22 M. Mulaj, J. Foley and M. Muschol, J. Am. Chem. Soc., 2014, 136, 8947-8956. 23 C. Galvagnion, J. W. P. Brown, M. M. Ouberai, P. Flagmeier, M. Vendruscolo, A. K. Buell, E. Sparr and C. M. Dobson, Proc. Natl. Acad. Sci. U. S. A., 2016, 113, 7065-7070.

24 P. Flagmeier, G. Meisl, M. Vendruscolo, T. P. Knowles, C. M. Dobson, A. K. Buell and C. Galvagnion, Proc. Natl. Acad. Sci. U. S. A., 2016, 113, 10328-10333.

25 J. W. P. Brown, A. K. Buell, T. C. T. Michaels, G. Meisl, J. Carozza, P. Flagmeier, M. Vendruscolo, T. P. J. Knowles, C. M. Dobson and C. Galvagnion, Sci. Rep., 2016, 6, 36010.

26 G. Taschenberger, J. Toloe, J. Tereshchenko, J. Akerboom, P. Wales, R. Benz, S. Becker, T. F. Outeiro, L. L. Looger, M. Bahr, M. Zweckstetter and S. Kugler, Ann. Neurol., 2013, 74, 109-118.

27 G. Yamin, L. A. Munishkina, M. A. Karymov, Y. L. Lyubchenko, V. N. Uversky and A. L. Fink, Biochemistry, 2005, 44, 9096-9107.

28 C. Roodveldt, A. Andersson, E. J. De Genst, A. Labrador-Garrido, A. K. Buell, C. M. Dobson, G. G. Tartaglia and M. Vendruscolo, Biochemistry, 2012, 51, 8771-8778.

29 G. M. Moriarty, M. P. Olson, T. B. Atieh, M. K. Janowska, S. D. Khare and J. Baum, J. Biol. Chem., 2017, 292, 16368-16379.

30 A. I. Sulatskaya, A. A. Maskevich, I. M. Kuznetsova, V. N. Uversky and K. K. Turoverov, PLoS One, 2010, 5, e15385.

31 S. a. Semerdzhiev, D. R. Dekker, V. Subramaniam and M. M. a. E. Claessens, ACS Nano, 2014, 5543-5551.

32 A. K. Buell, A. Dhulesia, D. A. White, T. P. J. Knowles, C. M. Dobson and M. E. Welland, Angew. Chem., Int. Ed., 2012, 51, 5247-5251.

33 G. Meisl, J. B. Kirkegaard, P. Arosio, T. C. T. Michaels, M. Vendruscolo, C. M. Dobson, S. Linse and T. P. J. Knowles, Nat. Protoc., 2016, 11, 252-272. 